EPJ Web of Conferences 41, 04017 (2013)

DOI: $10.1051 /$ epjconf/20134104017

(C) Owned by the authors, published by EDP Sciences, 2013

\title{
Surface Carrier Dynamics on Semiconductor Studied with Femtosecond Core-Level Photoelectron Spectroscopy Using Extreme Ultraviolet High-Order Harmonic Source
}

\author{
K. Oguri, T. Tsunoi, K. Kato, H. Nakano*, T. Nishikawa, H. Gotoh, K. Tateno, and T. Sogawa \\ NTT Basic Research Laboratories, Nippon Telegraph and Telephone Corporation, 3-1, Wakamiya, \\ Morinosato, Atsugi-shi, Kanagawa 243-0198, Japan \\ *Present address: Department of Electrical, Electronic and Computer Engineering Faculty of Science \\ and Engineering, Toyo University, 2100 Kujirai, Kawagoe-shi, Saitama 350-8585, Japan
}

\begin{abstract}
We have used a femtosecond time-resolved core-level surface PES system based on the 92-eV harmonic source to study the surface carrier dynamics that induces the transient SPV on semiconductor surfaces. We clarified the temporal evolution of the transient SPV characterized by the time of the photo-generated carrier separation and recombination. This result demonstrates the potential of this technique for clarifying the initial stage of the surface carrier dynamics after photoexcitation.
\end{abstract}

\section{Introduction}

The dynamic process of non-equilibrium surface charge carriers generated by irradiating semiconductor surfaces and interfaces with a light pulse has been of great interest since it plays an essential role in a wide variety of phenomena related to optoelectronic device physics and photochemistry [1]. In particular, surface carrier transport due to a pre-existing band bending in the space-charge region at semiconductor surfaces, which induces a transient surface photovoltage (SPV) by a photoexcitation on the surfaces, is an elementary process in various important surface applications such as photosensing, photovoltaics, and photocatalysis. Therefore, the surface carrier dynamics related to the transient SPV is one of the key factors that affect their response time or their conversion efficiency. Although the surface carrier processes has a fundamental time scale of from femtosecond to picosecond order, the SPV dynamics has mainly been measured on a subnanosecond to microsecond time scale with the core-level photoelectron spectroscopy (PES) using the combination of a pulse laser and a synchrotron radiation source [2, 3]. As a result, there is little experimental knowledge about the transient SPV dynamics on the fundamental time scale of the surface carrier.

Here, we present the study of ultrafast dynamics of the photo-generated carriers on undoped GaAs surface using a new PES technique with a high-order harmonic generation (HHG) source [4, 5]. We developed a femtosecond time-resolved core-level surface PES system based on the 59th harmonic source $(92 \mathrm{eV})$ generated by a $100-\mathrm{fs}$ laser pulse. Application of the $92-\mathrm{eV}$ harmonic pulse to PES has made it possible to realize ultrafast core-level photoelectron spectroscopy with a temporal resolution of approximately $100 \mathrm{fs}$ and an ultimate surface sensitivity on the scale of a few atomic layers. Using this system, we measured a temporal evolution of the transient SPV by detecting the energy shift of the Ga-3d core-level photoelectron peak induced by $1.5-\mathrm{eV}$ laser pulse

This is an Open Access article distributed under the terms of the Creative Commons Attribution License 2.0, which permits unrestricted use, distribution, and reproduction in any medium, provided the original work is properly cited. 
irradiation. The measurement revealed that the separation of the photo-generated carrier occurs less than $1 \mathrm{ps}$, and that the carrier recombination hardly proceeds at least up to 16 ps on GaAs (001) surface.

\section{Experimental setup}

The basic design of the femtosecond time-resolved core-level surface PES system is almost similar to that described in our previous study except for the pulse duration of the pump pulse[6]. Briefly, the system consists of a 100-fs Ti:sapphire laser system with a central wavelength of $790 \mathrm{~nm}$ and a repetition rate of $10 \mathrm{~Hz}$, an $\mathrm{HHG}$ vacuum chamber, a photoelectron energy analyser, and a sample preparation plus load-lock chamber. We used a Ne gas jet for $\mathrm{HHG}$ and a pair of $\mathrm{Mo} / \mathrm{Si}$ multilayer mirrors to select the 59th harmonic from the multiple harmonic peaks. Photoelectron spectra were measured with a 500-mm-long time-of-flight electron spectrometer. We used an undoped GaAs (001) substrate as the sample. The sample was chemically etched to remove a native oxidation layer from the GaAs surface and rinsed in $\mathrm{H}_{2} \mathrm{O}$. The sample was annealed at $400{ }^{\circ} \mathrm{C}$ for 15 min. Using the pump pulse with a photon energy of $1.5 \mathrm{eV}$, we almost matched the excitation energy to the direct band gap of GaAs in order to exclude the effect of hot carriers with much excess energy. All the measurements were performed at room temperature.

\section{Results and discussion}

Figure 1 shows typical transient changes in the photoelectron spectrum of the Ga-3d core-level peak at various delay times. The red and blue curves in Fig. 1 represent respectively the Ga-3d peaks with and without laser irradiation with an energy density of $0.1 \mathrm{~mJ} / \mathrm{cm}^{2}$. When the 59th harmonic pulse arrives at the sample earlier than the laser pulse, the two spectra are almost identical. At a time delay of 0 ps, i.e., when both beams overlap in time, the peak clearly shifts toward a higher binding energy. After that, the peak shift rapidly increases with the delay time, and then the magnitude of the peak shift hardly changes after a long time delay. The origin of the core-level peak shift is well known as the SPV effect, where photo-generated electron-hole pairs spatially separate according to the pre-existing band bending, resulting in the suppression of the band bending due to an additional

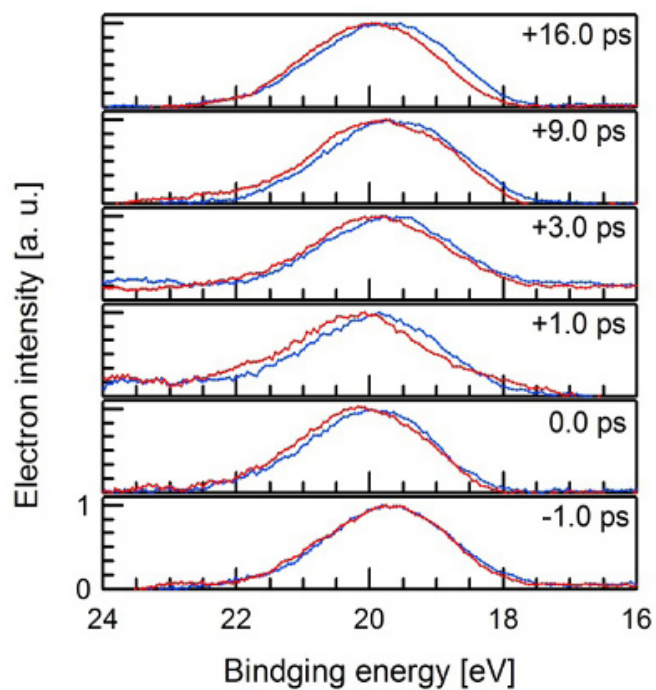

Fig. 1. Temporal evolution of the photoelectron energy spectrum of the Ga-3d core-level peak. Red and blue curves represent the peak with and without laser irradiation with an energy density of $0.1 \mathrm{~mJ} / \mathrm{cm}^{2}$, respectively. 
electric field formed by the separated electrons and holes [2]. The peak shift toward a higher binding energy indicates that the band bends upwards from the bulk to the surface, which is like n-type GaAs $[2,3]$.

We plotted the temporal evolution of the relative shift of the Ga-3d peak in Fig. 2. We can see that the shift rapidly increases from 0 to approximately $200 \mathrm{meV}$ at a delay time of from -1 to $1 \mathrm{ps,}$ and it then maintains almost the same shift at least up to a delay time of $16 \mathrm{ps}$. Since the rise time for the initial increase in the peak shift is slower than the pulse duration of the pump and probe pulses, it is likely to correspond to the separation time of the photo-generated electron-hole pairs. Since the length of the space-charge region is generally a few tens $\mathrm{nm}$, the separation time of less than 1 ps suggests that the photo-excited electron transports in the space-charge region with a speed near a saturation velocity of roughly $105 \mathrm{~m} / \mathrm{s}$ [7]. In addition, the fact that there is no clear reduction in the peak shift after a long time delay suggests that the surface carrier recombination time is much longer than the experimental value of $15 \mathrm{ps}$ in the pioneering report that measured p-type GaAs (110) using $3.1-\mathrm{eV}$ laser excitation with an energy density of $0.7 \mathrm{~mJ} / \mathrm{cm}^{2}$ [4]. This result indicates that the carrier recombination time is significantly affected by doping and excitation energy, and possibly surface orientation.

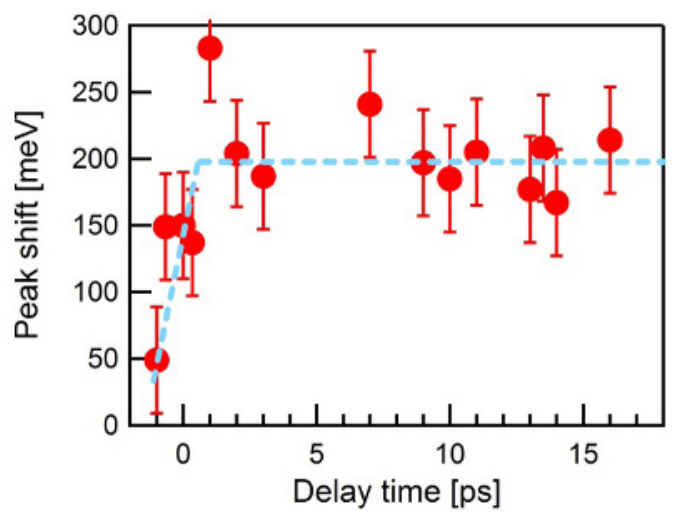

Fig. 2. The peak shift towards higher binding energy plodted as a function of delay time. The blue dotted line is an eye guide.

\section{Acknowledgments}

We thank Prof. Takeda and Prof. Katayama (Yokohama National University) for helpful discussions. This work was partly financed by a Grant-in-Aid for Scientific Research (No. 23310086) from the Ministry of Education, Culture, Sports, Science and Technology of Japan.

\section{References}

1. Y. Terada, S. Yoshida, O. Takeuchi, and H. Shigekawa, Nature Photon. 4, 869 (2010).

2. J. P. Long, H. R. Sadeghi, J. C. Rife, and M. N. Kabler, Phys. Rev. Lett. 64, 1158 (1990).

3. S. Tanaka, S. D. More, J. Murakami, M. Itoh, Y. Fujii, and M. Kamada, Phys. Rev. B 64, 155308 (2001).

4. R. Haight, and D. R. Peale, Rev. Sci. Instrum. 65, 1853 (1994).

5. P. Siffalovic, M. Drescher, and U. Heinzmann, Europhys. Lett. 60, 924 (2002).

6. K. Oguri, K. Kato, T. Nishikawa, H. Gotoh, K. Tateno, T. Sogawa and H. Nakano, Jpn. J. Appl. Phys. 51, 072401 (2012).

7. J. G. Ruch and G. S. Kino, Appl. Phys. Lett. 10, 40 (1967). 